\title{
Hunting a Type 2 fullerene nanodisc
}

\author{
M. da Cruz, ${ }^{\oplus}$ M. Tovar, ${ }^{\oplus}$ C. Figueiredo ${ }^{\oplus}$ and D. Sasaki
}

\begin{abstract}
A total coloring assigns colors to the vertices and edges of a graph without conflicts. The Total Coloring Conjecture is settled for cubic graphs, but not to all regular graphs nor to arbitrary planar graphs. We investigate the total coloring of fullerene nanodiscs, a subclass of cubic planar graphs with girth 5 arising in Chemistry, motivated by a conjecture about the nonexistence of a Type 2 cubic graph of girth at least 5 . We provide a combinatorial description and then a conformable coloring for small fullerene nanodiscs.
\end{abstract}

\section{Introduction}

A total coloring $C^{T}$ of a graph $G$ is a color assignment from set $E \cup V$, such that distinct colors are assigned to: every pair of vertices that are adjacent; all edges that are adjacent; and each vertex and its incident edges. Clearly, a total coloring of a graph $G$ requires at least $\Delta(G)+1$ colors, where $\Delta(G)$ is the maximum degree of $G$. A $k$-total coloring of a graph $G$ is a total coloring that uses a set of $k$ colors, and the graph is $k$ total colorable if it admits a $k$-total coloring. The total chromatic number of $G$ is the smallest natural $k$ for which $G$ admits a $k$-total coloring, and it

2000 AMS Subject Classification: 05C10, 05C15, 92E10.

Keywords and Phrases: Graph Theory, Total Coloring, Fullerenes, Nanodiscs.

This research was supported by CAPES, CNPq and FAPERJ. 
is denoted by $\chi^{\prime \prime}(G)$. Behzad and Vizing [2, 16] independently conjectured the Total Coloring Conjecture (TCC) that for any simple graph $G$, we have $\chi^{\prime \prime}(G) \leq \Delta(G)+2$. If $\chi^{\prime \prime}(G)=\Delta(G)+1$, then the graph is Type 1 ; if $\chi^{\prime \prime}(G)=\Delta(G)+2$, then the graph is Type 2 . The TCC is open for regular graphs, and has been verified for some particular classes of graphs. For cubic graphs that are graphs where every vertex has degree 3 , the TCC has already been settled [8], but it is not yet settled for all planar graphs [10]. Deciding whether a cubic bipartite graph is Type 1 is NP-complete [13].

We investigate the total coloring problem considering cubic planar graphs with large girth that model chemical structures: the fullerene nanodiscs. The length of the shortest cycle in a graph is called girth and has been considered for total coloring planar graphs [3]. Our goal is to study a conjecture proposed by Brinkmann, Preissmann and Sasaki [4] which states that every Type 2 cubic graph has girth less than 5, and suggests that the girth of a graph is a relevant parameter in the study of total coloring. The hunting of special Type 2 cubic graphs has been considered for nonplanar graphs as well [15]. We contribute by giving a combinatorial description of the small fullerene nanodiscs, and then by showing that they are conformable, a necessary step towards proving that they are Type 1.

\section{$2 \quad$ Fullerene graphs}

To motivate the choice of the studied graph class, we first give a historical account of the discovery of the carbon molecule which can be modeled through a special cubic planar graph of girth 5, and then establish a combinatorial description of our target class.

\subsection{Fullerene: A graph class modeling a molecule}

In attempt to understand the spectroscopy data obtained from astronomical objects as giant red carbon stars, which indicates the formation of long chains of carbon atoms, Harold Kroto and coworkers [9] performed a series of experiments vaporizing and cooling highly pure carbon samples 
discovering a new carbon allotrope highly symmetrical stable molecule, composed of 60 tertiary carbon atoms. As the only way to maintain this amount of carbon atoms stable, Richard Smalley proposed a spheroid structure similar to a geodesic dome built by the Architect Buckminster Fuller, with 32 faces, being 20 hexagonal and 12 pentagonal (see Figure 1 ) later named by them as " $C_{60}$ buckminsterfullerene", or simply "buckyball" since the molecule structure is shaped like a soccer ball. For this contribution Harold Kroto, Robert Curl and Richard Smalley earned the 1996 Chemistry Nobel Prize. Fullerene molecules are widely studied by different branches of science, from medicine to mathematics. These molecules are supposed to contribute to transport chemotherapy, antibiotics or antioxidant agents, and are released in contact with deficient cells [14]. At the end of the 1980s, other carbon allotrope molecules with similar spatial structure to the $C_{60}$ were reported to be called fullerene molecules, defined as polyhedral carbon cages in which $s p^{2}$-carbons are directly bonded to three neighbors in an arrangement of five- and six-membered rings [1].

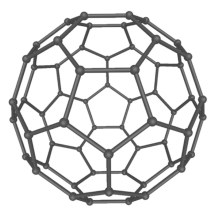

(a)

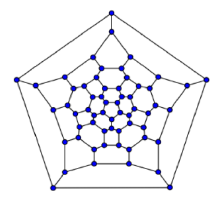

(b)

Figure 1: molecular structure (a) and fullerene graph (b) of $C_{60}$.

Each fullerene molecule can be described as a planar graph in which the atoms and the bonds are represented by the vertices and edges of the graph, respectively, preserving the geometric properties of the original fullerene molecule. Thus, we define a fullerene graph as cubic, planar, 3connected graph whose faces are pentagonal or hexagonal (see Figure 1). The famous Euler's formula for connected planar graphs $n+f-m=2$ relates the number $f$ of faces, the number $m$ of edges and the number $n$ of vertices, and implies that every fullerene graph must contain exactly 
12 pentagons, and that the smallest fullerene graph is the well known dodecahedron with 20 vertices where all faces are pentagons [1].

\section{$2.2 \quad$ Fullerene nanodiscs}

We shall consider a particular family of fullerene graphs. According to the 3-dimensional structure, the fullerene nanodiscs, or nanodiscs of radius $r \geq 2$, are structures composed of two identical flat covers connected by a strip along their borders. While in the nanodisc covers there are only hexagonal faces, in the connecting strip, besides the hexagonal faces, additional 12 pentagonal faces are arranged. Please refer to Figure 2 where the smallest fullerene nanodisc graphs are depicted. In each fullerene nanodisc graph, we highlight in the connecting strip the 12 pentagons.

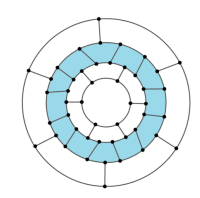

$D_{2}$

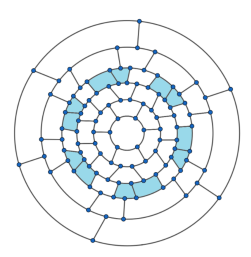

$D_{3}$

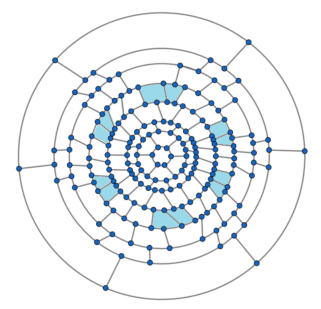

$D_{4}$

Figure 2: The smallest fullerene nanodisc graphs.

A nanodisc graph of radius $r \geq 2$, denoted by $D_{r}$, has its faces arranged into layers, one layer next the nearest previous layer starting from an hexagonal cover until we reach the other hexagonal cover [12]. The sequence $\{1,6,12, \ldots, 6(r-1), 6 r, 6(r-1), \ldots, 12,6,1\}$ provides the amount of faces on each layer of the nanodisc graph $D_{r}$. Note that there is an odd number of $2 r+1$ layers, and we call the layer with $6 r$ faces the central layer. For $D_{2}$ the layer sequence is $\{1,6,12,6,1\}$, for $D_{3}$ is $\{1,6,12,18,12,6,1\}$ and for $D_{4}$ is $\{1,6,12,18,24,18,12,6,1\}$ (see Figure 2). The auxiliary cycle sequence provides the sizes of the auxiliary cycles that define the layers $\left\{C_{6}, C_{18}, \ldots, C_{12 r-6}, C_{12 r-6}, \ldots, C_{18}, C_{6}\right\}$. For example, for $D_{2}$ the cycle 
sequence is $\left\{C_{6}, C_{18}, C_{18}, C_{6}\right\}$, for $D_{3}$ is $\left\{C_{6}, C_{18}, C_{30}, C_{30}, C_{18}, C_{6}\right\}$, and for $D_{4}$ is $\left\{C_{6}, C_{18}, C_{30}, C_{42}, C_{42}, C_{30}, C_{18}, C_{6}\right\}$ (see Figure 2). A nanodisc graph $D_{r}$ contains $12 r \times r$ vertices and $18 r \times r$ edges.

The 12 pentagonal faces are distributed in the central layer among its $6 r$ faces with the other $(6 r-12)$ hexagonal faces. This is the key property of fullerene nanodiscs [12]. Note that the central layer is defined by two auxiliary cycles, each of size $12 r-6$. The 5 vertices of each pentagon are partitioned such that 3 vertices appear consecutively in one cycle and 2 vertices appear consecutively in the other cycle. We say that two pentagons in the central layer are partitioned in the same way if each pentagon has 3 vertices in the same cycle $C_{12 r-6}$. For $r \geq 2$, in a $D_{r}$, we may have choice to distribute and to partition the 12 pentagonal faces. Our first result imposes restrictions to the way the pentagons are partitioned in the central layer of a nanodisc graph and ensures that there is a unique $D_{2}$.

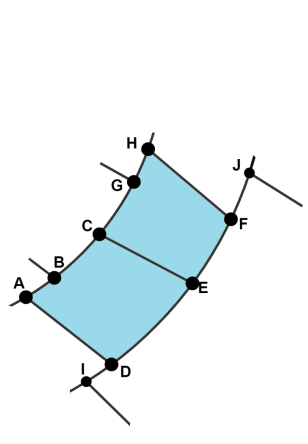

(a)

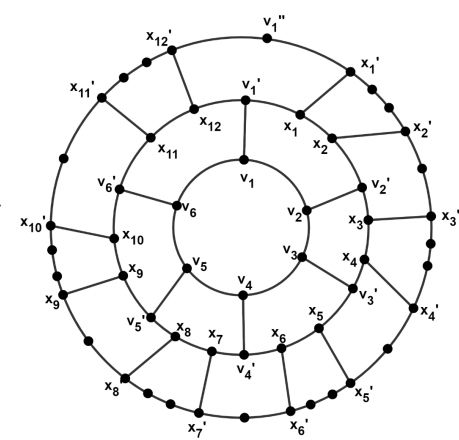

(b)

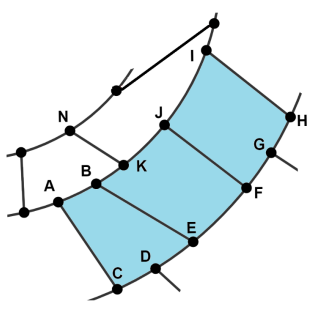

(c)

Figure 3: (a) Two consecutive pentagons partitioned in the same way; (b) Labels of the vertices in three cycles of $D_{3}$; (c) Three consecutive pentagons in the central layer of $D_{3}$.

Proposition 2.1. A nanodisc $D_{r}, r \geq 2$, cannot have two consecutive pentagons in the central layer partitioned in the same way.

Proof. In Figure 3(a), we show two pentagons (namely $A B C E D$ and $C G H F E$ ) partitioned in the same way and arranged side by side in the 
central layer of $D_{r}$, so that vertices $A, B, C, G$ and $H$ belong to the same auxiliary cycle and the other vertices $D, E$ and $F$ belong to the other.

Consider the layer adjacent to the central layer, where one of its defining cycles contains vertices $D, E, F$. By considering vertices $I$ and $J$ adjacent respectively to $D$ and $F$ in the cycle $C_{12 r-6}$, we find a path containing five consecutive vertices $I, D, E, F, J$ in the cycle, leading to a contradiction that $I, D, E, F, J$ must lie in a forbidden face.

Observe that there are two ways of partitioning a hexagon in a layer defined by auxiliary cycles $C$ and $C^{\prime}$. We may place 3 vertices of the hexagon in each auxiliary cycle, or place 4 vertices of the hexagon in one auxiliary cycle say $C$ and the other 2 vertices in $C^{\prime}$. Our next results consider $D_{3}$ and impose restrictions to the way the hexagons are partitioned and the pentagons are distributed, and ensure that there is a unique $D_{3}$.

Proposition 2.2. In $D_{3}$, the layers consisting of 12 hexagons cannot have two consecutive hexagons partitioned in the same way.

Proof. Please refer to Figure $3(b)$. Label as $v_{i}$ the vertices of the inner $C_{6}$ of $D_{3}$ that define radial edges with vertices $v_{i}^{\prime}$ in the next cycle $C_{18}$. The inner $C_{6}$ and the next $C_{18}$ build a layer consisting of 6 hexagons. In the $C_{18}$, we must distribute 12 additional vertices $x_{i}$ in pairs, between the $v_{i}^{\prime}$, as follows: $x_{1}$ and $x_{2}$ between $v_{1}^{\prime}$ and $v_{2}^{\prime}, x_{3}$ and $x_{4}$ between $v_{2}^{\prime}$ and $v_{3}^{\prime}, \ldots$, and $x_{11}$ and $x_{12}$ between $v_{6}^{\prime}$ and $v_{1}^{\prime}$. All 6 hexagons in the inner layer have 2 vertices appearing consecutively in the inner cycle $C_{6}$ and 4 vertices appearing consecutively in the next cycle $C_{18}$. The vertices $x_{i}$ define radial edges with 12 vertices $x_{i}^{\prime}$ in the next cycle $C_{30}$. We have a hexagon defined by three vertices $x_{12}, v_{1}^{\prime}, x_{1}$ in the $C_{18}$, and vertices $x_{12}^{\prime}, x_{1}^{\prime}$, plus $v_{1}^{\prime \prime}$ between them, in the $C_{30}$. The two hexagons that lie on each side of the hexagon must be partitioned such that 2 vertices appear consecutively in the $C_{18}$ and 4 vertices appear consecutively in the $C_{30}$.

Proposition 2.3. In $D_{3}$, the central layer cannot have three consecutive pentagons. 
Proof. From Proposition 2.1, we cannot have two consecutive pentagons in the central layer partitioned in the same way, so we have in Figure $3(c)$ three consecutive pentagons so that between two pentagons partitioned in the same way, there is a pentagon partitioned in a different way.

The central layer of the $D_{3}$ is defined by two cycles $C_{30}$. Consider in Figure $3(c)$ five vertices $A, B, K, J$ and $I$ that are consecutive in a $C_{30}$, and observe that among them only vertex $K$ is adjacent to a vertex $N$ in a $C_{18}$. So the two consecutive hexagons, of the layer defined by $C_{30}$ and a $C_{18}$, containing vertices $A, B, K, J$ and $I$, and additionally the vertex $N$, must be partitioned in the same way, which contradicts Proposition 2.2 .

In the central layer of $D_{3}$, we have 12 pentagons distributed among 6 hexagons. By Proposition 2.1, we cannot have two consecutive pentagons partitioned in the same way. By Proposition 2.3, we cannot have three consecutive pentagons. So, among the 6 hexagons, the 12 pentagons must appear in pairs of consecutive pentagons where each pair is not partitioned in the same way. See the unique $D_{3}$ in Figure 2 .

\section{Conformable coloring of fullerene nanodiscs}

The smallest Type 2 cubic graph is $K_{4}$, and another known Type 2 cubic graph is the generalized Petersen graph $G(5,1)$ [5], and both have squares or triangles, as well as all Type 2 cubic graphs found so far [7]. The evidence found in the work of Brinkmann et al. [4] suggests the conjecture that every Type 2 cubic graph has a girth less than 5 , and searches were started for Type 1 and Type 2 fullerene graphs, which are graphs with girth 5, but so far only Type 1 fullerene graphs have been obtained [11.

A necessary step towards proving that a cubic graph is Type 1 is to define a 4 -vertex coloring where the cardinality of each vertex color class has the same parity when compared to the cardinality of the entire vertex set. Such special vertex coloring is called conformable [5]. From the unicity established in Subsection 2.2 for $D_{2}$ and $D_{3}$, we may present in Figure 4; (a) a 4-total coloring for $D_{2}$ [6], and (b) an optimal 3-vertex 
coloring for $D_{3}$ that satisfies the requirement to be a conformable 4-vertex coloring since each of the 4 color classes has an even number of elements.

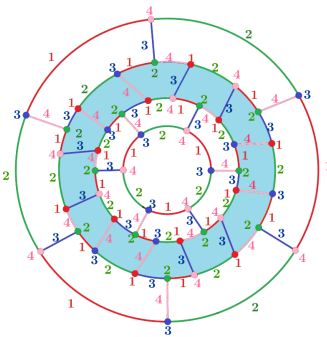

(a)

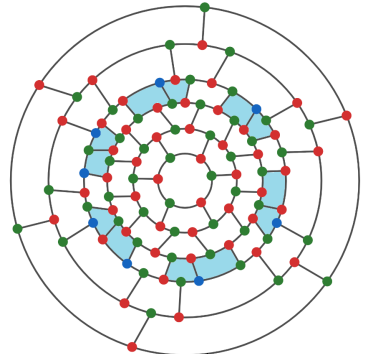

(b)

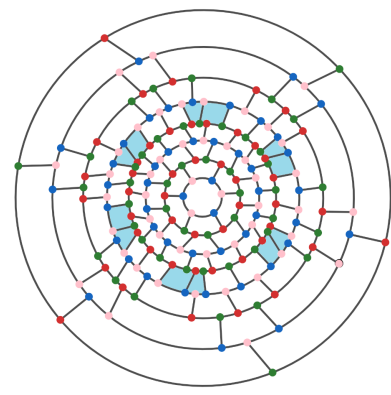

(c)

Figure 4: (a) A 4-total coloring of $D_{2} ;(b) \mathrm{A} 3$-vertex coloring that is a 4-conformable coloring of $D_{3} ;(c)$ A 4-conformable coloring of $D_{4}$.

Another strategy is to take advantage that the auxiliary cycles have even length and color alternately with colors 1 and 2 the cycle $C_{6}$ defining the inner layer, with colors 3 and 4 the next cycle $C_{18}$, and so on. The strategy does not rely on the unicity of $D_{r}$, for $r>3$, and defines a conformable 4 -vertex coloring for even radius as proved next. Our current goal is to generalize the 3 -vertex coloring that is a 4-conformable coloring of $D_{3}$ presented in Figure 4 (b) to an arbitrary nanodisc of odd radius.

Theorem 3.1. Every nanodisc with even radius admits a conformable 4-vertex coloring.

Proof. Please refer to Figure $4(c)$. In a $D_{r}$ with even $r$, consider the 4vertex coloring that gives colors 1 and 2 to the outer cycle $C_{6}$, colors 3 and 4 to the next cycle $C_{18}$, until we reach the central layer, where colors 3 and 4 are given to cycle $C_{12 r-6}$ and colors 1 and 2 are given to the next cycle $C_{12 r-6}$, continue in this fashion until colors 3 and 4 are given to the inner cycle $C_{6}$. Note that each cycle provides an odd number of colored vertices with the same color, and that every color class has the same number of vertices and that this number is even, given by $3 r \times r$. 


\section{Acknowledgment}

We are grateful to two reviewers for their careful reading with many suggestions which contributed to the presentation of the extended abstract.

\section{References}

[1] K. Ala'a, J. P. Hare, H. Kroto, and R. Taylor, Isolation, separation and characterisation of the fullerenes $C_{60}$ and $C_{70}$ : the third form of carbon, Journal of the Chemical Society, Chemical Communications 20 (1990), pp. 1423-1425.

[2] M. Behzad, Graphs and Their Chromatic Numbers, PhD Thesis, Michigan University (1965).

[3] O. V. Borodin, A. V. Kostochka and D. R. Woodall, Total colourings of planar graphs with large girth, European Journal of Combinatorics 19 (1998), 19-24.

[4] G. Brinkmann, M. Preissmann and D. Sasaki, Snarks with total chromatic number 5, Discrete Mathematics and Theoretical Computer Science 17 (2015), pp. 369-382.

[5] A. G. Chetwynd and A. J. W. Hilton, Some refinements of the total chromatic number conjecture, Congressus Numerantium 66 (1988), pp. 195-216.

[6] M. M. F. da Cruz, D. Sasaki, and M. V. T. Costa, Coloração Total de Nanodiscos de Fulerenos, Cadernos do IME-Série Matemática 15 (2020), pp. 34-53.

[7] S. Dantas, C. M. H. de Figueiredo, G. Mazzuoccolo, M. Preissmann, V. F. dos Santos, and D. Sasaki, On the total coloring of generalized Petersen graphs, Discrete Mathematics 339 (2016) pp. 1471-1475. 
[8] A. V. Kostochka, The total chromatic number of any multigraph with maximum degree five is at most seven, Discrete Mathematics 162 (1996), pp. 199-214.

[9] H. W. Kroto, J. R. Heath, S. C. O'Brien, R. F. Curl, and R. E. Smalley, $C_{60}$ : Buckminsterfullerene, Nature 318 (1985), pp. 162-163.

[10] M. Leidner, A larger family of planar graphs that satisfy the total coloring conjecture, Graphs and Combinatorics 30 (2014), pp. 377388.

[11] D. S. Nicodemos, Diâmetro de Grafos fulerenos e Transversalidade de Ciclos Ímpares de Fuleróides-(3,4,5,6), Tese de doutorado. Rio de Janeiro: COPPE/UFRJ (2017).

[12] D. Nicodemos and M. Stehlík, Fullerene graphs of small diameter, MATCH Communications in Mathematical and in Computer Chemistry 77 (2017), pp. 725-728.

[13] A. Sánchez-Arroyo, Determining the total colouring number is NPhard, Discrete Mathematics 78 (1989), pp. 315-319.

[14] L. J. Santos, G. P. Rocha, R. B. Alves, and R. P. de Freitas, Fulereno [C60]: química e aplicações, Química Nova 33 (2010), pp. 680-693.

[15] D. Sasaki, S. Dantas, C. M. H. de Figueiredo, M. Preissmann, The hunting of a snark with total chromatic number 5, Discrete Applied Mathematics 164 (2014), pp. 470-481.

[16] V. Vizing, On an estimate of the chromatic class of a p-graph, Discret Analiz 3 (1964), pp. 25-30. 

M. da Cruz
D. Sasaki

Rio de Janeiro Federal University Rio de Janeiro State University (COPPE/UFRJ) (IME/UERJ)

Rio de Janeiro, Brazil. Rio de Janeiro, Brazil.

mmartins@cos.ufrj.br diana.sasaki@ime.uerj.br

M. Tovar

C. Figueiredo

Rio de Janeiro State University Rio de Janeiro Federal University (IME/UERJ) (COPPE/UFRJ)

Rio de Janeiro, Brazil. Rio de Janeiro, Brazil. marcus.tovar@ime.uerj.br celina@cos.ufrj.br 